\title{
Electroencephalogram (EEG) stress analysis on alpha/beta ratio and theta/beta ratio
}

\author{
Tee Yi Wen, Siti Armiza Mohd Aris \\ Razak Faculty of Technology and Informatics, Universiti Teknologi Malaysia, Malaysia
}

\begin{tabular}{|c|c|}
\hline Article Info & ABSTRACT \\
\hline Article history: & This paper presents an analysis of stress feature using the power ratio of \\
\hline Received May 18, 2019 & $\begin{array}{l}\text { frequency bands including Alpha to Beta and Theta to Beta. In this study, } \\
\text { electroencephalography (EEG) acquisition tool was utilized to collect brain }\end{array}$ \\
\hline Revised Jul 20, 2019 & signals from 40 subjects and objectively reflected stress features induced by \\
\hline Accepted Aug 7, 2019 & $\begin{array}{l}\text { virtual reality (VR) technology. The EEG signals were analyzed using } \\
\text { Welch's fast Fourier transform (FFT) to extract power spectral density (PSD) }\end{array}$ \\
\hline Keywords: & $\begin{array}{l}\text { features which represented the power of a signal distributed over a range of } \\
\text { frequencies. Slow wave versus fast wave (SW/FW) of EEG has been studied }\end{array}$ \\
\hline $\begin{array}{l}\text { Electroencephalography } \\
\text { Power ratio } \\
\text { Power spectral density }\end{array}$ & $\begin{array}{l}\text { to discriminate stress from resting baseline. The results showed the } \\
\text { Alpha/Beta ratio and Theta/Beta ratio are negatively correlated with stress } \\
\text { and indicated that the power ratios can discriminate the data characteristics of } \\
\text { brainwaves for stress assessment. }\end{array}$ \\
\hline
\end{tabular}

Copyright (c) 2020 Institute of Advanced Engineering and Science. All rights reserved.

\section{Corresponding Author:}

Siti Armiza Mohd Aris,

Razak Faculty of Technology and Informatics,

Universiti Teknologi Malaysia,

Jalan Sultan Yahya Petra, 54100 Kuala Lumpur, Malaysia.

Email: armiza.kl@utm.my

\section{INTRODUCTION}

Stress in the long run contributes detrimental impacts for body and mental health. When stress stimulus is perceived, the brain activates an adaptive response to stress through the releasing of different hormones to increase the mobilization of energy stores for the adaption to short-term threatening situations. The stress response is adequate in the short-term but long-term stress situations may upset the equilibrium through physiological mechanism as in the immune system and lead to diverse diseases [1-2] such as cardiovascular diseases. The activation accompanied with the changes in behaviors like physical activity and eating habit leads to visceral fat accumulation and brings as a consequence of obesity and diabetes [3]. Furthermore, the changes of internal biological processes also cause mental health problems such as depression [4]. Hence, stress monitoring is crucial for early diagnosis and to prevent possible illnesses.

Various psychological tests have been devised in research and clinical practice for the purpose to obtain statistically useful information and measure stress level [5-6]. The assessments involve self-report or clinician-rated by using subjective perceptions and estimations to extract specific information on cognitive, emotional or behavioural stress responses. However, these methods are subjective and not sensitive enough to capture subtle patterns of mental state. Previous studies have found that subjective self-reported stress was insufficiently reflected by respective physiological parameters of the stress measurement [7-8]. Hence, physiological parameters served as an additional fairly objective and straightforward way to measure stress.

Electroencephalogram (EEG) tools have been introduced for stress assessment due to its ability to capture and interpret the changes of brain activity under different situations. The high temporal resolution provided by EEG is required to accurately record quick changes of electrical activity at a millisecond time scale. Moreover, EEG is affordable for clinical and research laboratories and easy to use. The combination of 
EEG and signal analysis methods empowers the investigation of neurological disorders [9]. Researchers have also analysed different kinds of human brain states. For instances, observe brain dominance [10], classify IQ levels [11] and human emotions [12]. In addition, EEG tools have demonstrated the use of brain signals to distinguish stress level which is impactful [13] with the capability of detecting and quantifying individual's stress level in an efficient manner merely through EEG signal.

In the work of EEG-based stress recognition, the power spectrum contained in the signals are often extracted as features [14-15] by using Fourier transform methods [16]. The oscillatory signals in time series data can be identified through power spectral density (PSD) because it represents the power of a signal distributed over a range of frequencies and each of the frequency band represents a state of the person [17]. The four basic frequency bands namely Delta $(0.5-4 \mathrm{~Hz})$, Theta $(4-8 \mathrm{~Hz})$, Alpha $(8-13 \mathrm{~Hz})$ and Beta (13-30Hz). Delta's waves tend to occur during deep and unconscious sleep. Theta is a common state for the brain and occurs whenever a person is in light sleep or quiet focus. Meanwhile, Alpha indicates the state of consciousness and happens during periods of relaxation with eyes closed but not sleep. Following this, Beta waves are observed during the state of normal consciousness, alertness or active concentration.

Stress pattern can be demonstrated by an increase of power in Beta band and decrease of power in Alpha band [18] on prefrontal cortex (PFC) [19]. Stress exposure causes effects on the regions of PFC functioning and it is recognized as the brain region susceptive to stress [20]. The ratio of slow wave over fast wave $(\mathrm{SW} / \mathrm{FW})$ of EEG is one of the techniques used by researchers to reflect the cortical control function over subcortical. Alpha/Beta ratio is used to relate the two most relevant frequencies in order to study the evolution of individual's cognitive state such as mental attentiveness [21] and mental fatigue [22]. Besides that, Theta/Beta ratio is initially introduced as an EEG marker for the use in attention deficit/hyperactivity disorder (AD/HD) diagnosis in term of arousal deficit [23]. However, subsequent studies have linked this metric to various aspect of executive cognitive performances in healthy individuals [24].

This paper presents the utilization of EEG tool in conjunction with signal processing technique to analyze stress feature in term of Alpha/Beta ratio and Theta/Beta ratio. The aim of this paper is to further investigate and predict the two ratios based on the PFC as potentially useful features for stress. Firstly, the EEG signals are pre-processed and further divided into Alpha, Beta, Theta and Delta frequency bands. The PSD contained in the four frequency bands are extracted using fast Fourier transform (FFT). Then, the PSD of Alpha, Beta and Theta frequency bands are averaged to calculate the power ratios (Alpha/Beta and Theta/Beta) to unveil and determine the relationship between the ratio and stress. Experimental results show that the values of Alpha/Beta ratio and Theta/Beta ratio decreased after exposed to the stress stimulus which denote more stressful state. This further verifies the validity and feasibility of the power ratios as stress features for the development of stress detection system.

\section{RESEARCH METHOD}

The experiment stage is divided into data collection, signal pre-processing, feature extraction and power ratio calculation. EEG signals were first accumulated followed by data pre-processing. Next, bands of specific frequencies were extracted from the pre-processed data. Subsequently, suitable features were extracted and selected to be analysed.

\subsection{Data Collection}

The are 26 male and 14 female subjects aged ranging from 19 to 38 years old have participated in this experiment. All the subjects are the undergraduate and postgraduate students from Universiti Teknologi Malaysia, Kuala Lumpur. They were in healthy conditions and were not under the influence of medicine and drugs prior to the experiment. For the stimulus, VR video was utilized as stress elicitation and delivered by using Samsung Gear VR device. VR is a form of technology which a three-dimensional (3D) world is created by computer and the realistic virtual environment empowers users to have better immersion and presence experience. In the work of [25], stress stimuli were generated by combining the arithmetic tasks with VR device. The subjects produced high stress scores on one of the VR environment which was a dingy room with frightening background sound. To obtain suitable fearful and stressful scene, YouTube website and video applications from Oculus were investigated. Finally, virtual roller coaster ride was chosen. Figure 1 shows the roller coaster in a post-apocalyptic world where the technological civilization has collapsed and overran by vampire-like beings who were infected by a highly contagious virus. This stimulus was used to induce the subjects' stress response for the analysis of stress state from brain activity. Before using the VR, resting state of the subjects was obtained as baseline for analysing the relax state and further distinguish the stress state. 


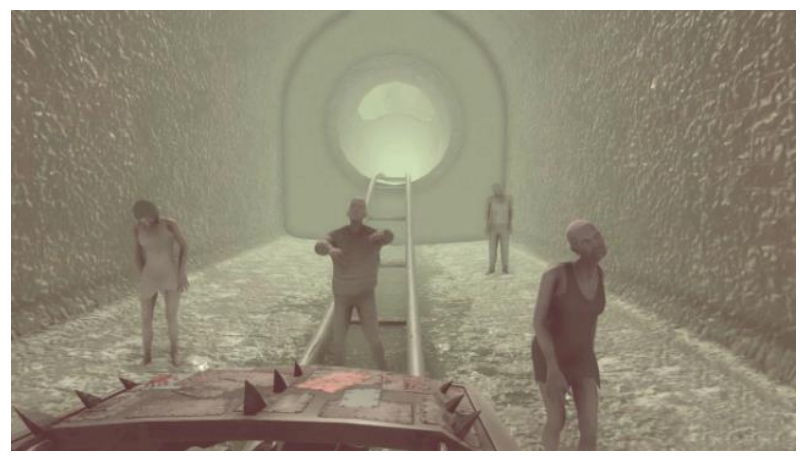

Figure 1. Virtual roller coaster ride in the post-apocalyptic world overran by vampires

The EEG signals were acquired by using EEG amplifier called g-MOBIlab connected to five pieces of bipolar gold-plated EEG electrodes. The location of the electrodes were fixed at the points chosen based on the international standard 10-20 electrode placement system. As shown in the Figure 2, Fp1, Fp2 and Fpz (ground) were denoted at prefrontal area of brain region (forehead area). Fp1 was used for the left side of the forehead and Fp2 for the right side of the forehead, connected to Channel 1 and Channel 2 respectively. Meanwhile, both A1 and A2 were attached to the earlobes for reference points. The brain signals were recorded using g-MOBIlab and transferred wirelessly to the computer using Simulink models integrated with MATLAB. The impedance of electrodes was measured below $5 \mathrm{k} \Omega$ where the EEG signals were sampled at sampling rate of $256 \mathrm{~Hz}$ and stored for offline analysis.

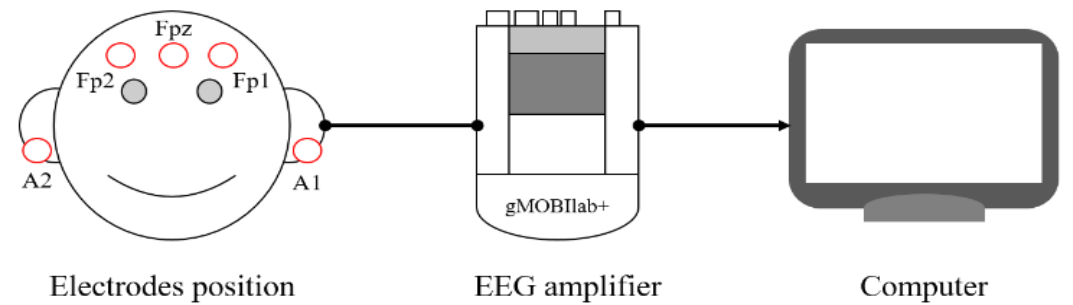

Figure 2. EEG measurement setup

Before starting the EEG recording, a brief introduction and instructions were given to the subjects to make them familiar with the experimental tasks. The subjects were required to fill in the demographic form as well to sign a consent form for the agreement to participate in the research. The subjects were seated in a quiet room with temperature about $22^{\circ} \mathrm{C}$ and sit comfortably on a chair with relaxing position. The subjects were also asked to minimize head movement as much as possible throughout the entire experiment in order to reduce unwanted noise. The subjects' forehead and both earlobes were cleaned with non-abrasive alcohol to remove dirt and dead cells to ensure a proper connections. In order to make the gold electrodes stick well onto the skin and further reduce the impedance on the sensors, the electrodes were filled with conductive paste and then placed on the forehead, both earlobes, left and right side of the forehead. The duration for whole experiment procedure was approximately 1 hour. The EEG signals were collected in two different sessions for resting baseline and stress session. Initially, the subjects were given 5 to 10 minutes to relax where they need to close their eyes but not sleep. The EEG signals for this relax state were recorded for 3 minutes to obtain baseline and stabilize the signal. After done the recording, the subjects wore Samsung Gear VR device to ride on a virtual roller coaster for 4 minutes. The EEG signals recording for another 3 minutes was performed immediately after using VR to obtain the stressful brain state.

\subsection{Signal Pre-Processing}

Pre-processing of EEG signals such as notch filter was performed using Simulink prior to converting the signals into desired file format. The notch filter was applied to remove $50 \mathrm{~Hz}$ noise from power cable line. Then, the recorded EEG signals were imported in MAT file format to be used for data analysis in MATLAB. Following this, artifacts removal and band-pass filter were included in this step. The artifacts can be removed by setting a threshold value below than $-100 \mu \mathrm{V}$ and greater than $100 \mu \mathrm{V}$ to keep 
meaningful and informatics signal. The pre-processed EEG signal is then decomposed into four sub-bands using band-pass filter. The Hamming windowing function was used to design the band-pass filter to cut off unwanted frequency and keep the required frequency from $0.5 \mathrm{~Hz}$ to $30 \mathrm{~Hz}$ including Delta $(0.5-4 \mathrm{~Hz})$, Theta $(4-8 \mathrm{~Hz})$, Alpha $(8-13 \mathrm{~Hz})$ and Beta $(13-30 \mathrm{~Hz})$ frequency bands. The design of band-pass filter with windowing function lessen the effects of leakage prior to performing Fourier-related transforms and enhance the ability to extract spectral data from signals. The default rectangular window is poor in noise suppression which in result the leakage amounts of spectral information from Fourier-related transforms showing up at the wrong frequencies because some energies of the signal has leaked out from the true frequency into adjacent frequencies. Hamming window functions with lower side lobes can exhibit less leakage [26].

\subsection{Feature Extraction}

FFT is a mathematic computational tool with powerful reversible mapping operation that is used to convert a signal from time domain to frequency domain. The PSD features of the artifact-free EEG signals can be estimated with the periodogram computed by FFT algorithm. However, the periodogram suffers from large variance and poor statistical accuracy. Welch provided a modified method of periodogram averaging which brings the result of decreasing the variance of the PSD. The procedures involve dividing the time series data into segments, possibly overlapping, then performing FFT, computing the magnitude of each segments and averaging these spectra. In this study, 1 minute were chosen as sample from the 3 minutes EEG signal and split into 12 equal segments with the window length of 5 seconds each. Time sequence of each 1 minute sample was divided into 50\% overlapping 12 segments. FFT was used to compute each of these segments and average the power of the FFT coefficients for all the overlapping segments. This computation is repeated for all frequency bands and finally the PSD $(\mu \mathrm{V} 2)$ values of Delta, Theta, Alpha and Beta were yielded.

The PSD according to Welch is expressed by [27]:

$$
P_{d}(f)=\frac{1}{M U}\left|\sum_{n=0}^{M-1} x_{d}(n) w(n) e^{-j 2 \pi f n}\right|^{2}
$$

Let $x_{d}(n)$ be the sequence, where $d=1,2,3, \ldots, L$ are the signal intervals and each interval length is $M . U$ is the normalization factor for the power in the window function while $w(n)$ is the windowed data, that is:

$$
U=\frac{1}{M} \sum_{n=0}^{M-1}|w(n)|^{2}
$$

The Welch power spectrum is the average over these modified periodograms can be represented as:

$$
P W e l c h(f)=\frac{1}{L} \sum_{i=0}^{L-1} P_{d}(f)
$$

Where PWelch is the periodogram of the EEG signal of each interval.

\subsection{Power Ratio}

The computed PSD of each frequency band was averaged and further used to calculate the power ratio by dividing absolute power of one frequency band by absolute power of another frequency band. In this study, the frequency bands of interest were Alpha, Beta and Theta. The two power ratios of interest were Alpha to Beta (Alpha/Beta ratio) and Theta to Beta (Theta/Beta ratio). Delta activity reflected deep sleeping state of a person and was not expected to show high activity during the stressful condition so it was not investigated in this study. The PSD of Alpha was divided by PSD of Beta in order to get Alpha/Beta ratio. Subsequently, the ratio value of the resting baseline and stress session was observed to study the difference before and after the stimulus. The procedures as well applied to calculate Theta/Beta ratio and distinguish between relax and stress state. Figure 3 represents the flow of data collection, signal pre-processing, feature extraction and power ratio calculation. 


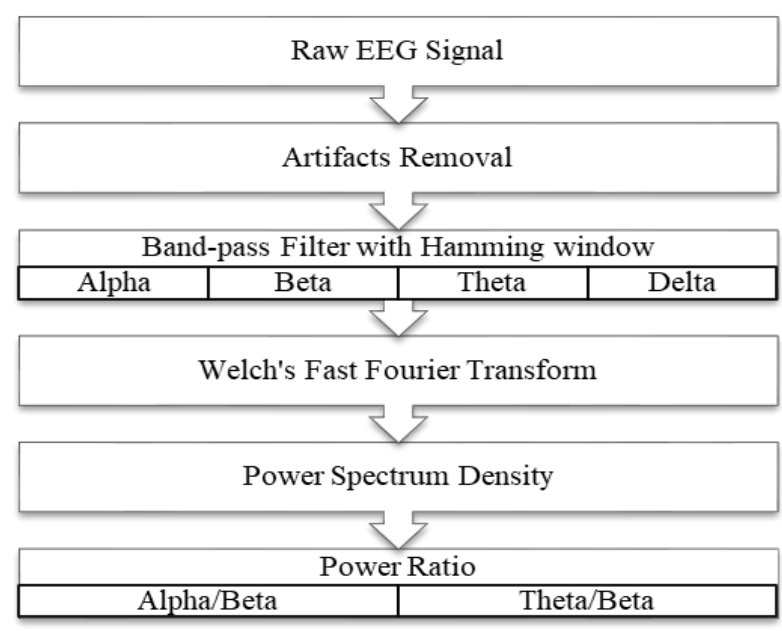

Figure 3. The overall flow of the EEG signal processing

\section{RESULTS AND ANALYSIS}

Above specified data pre-processing, feature extraction and power ratio calculation gave the results of stress pattern in the value of Alpha/Beta ratio and Theta/Beta ratio.

\subsection{Alpha/Beta Ratio}

Figure 4 shows the value of Alpha/Beta ratio is decreased in stress condition, which indicates Alpha activity is decreased, appears as an increased relative Beta activity. The smaller value of the ratio means the greater percentage of Beta activity [28]. Alpha oscillations emerge when the brain is relaxed meanwhile Beta oscillations appear when the brain is in active and stressful state. In previous studies, higher Alpha/Beta ratio reflecting increased Alpha power and/or decreased Beta power, were associated with a decreased level of alertness or arousal indicating higher fatigue level [22]. Besides that, another study reported that higher engagement in cognitive processes during decision making results in the declining value of Alpha/Beta ratio which informed less relaxed [29]. In Figure 4, stress condition results in lower ratio signifies a lower level of relaxation. Consider the resting baseline the less stressing condition, VR stimulus as the more stressing one, a negative correlation between stress and the Alpha/Beta ratio is unveiled. The brain activity before and after the stimulus can be reflected by the ratio of the power of Alpha to Beta frequency band. The differences in Alpha/Beta ratio shown in the Figure 4 confirmed between groups imply that the ratio is sensitive to minor changes or small differences in EEG stress feature. With the analysis of the Alpha/Beta ratio, it is possible to observe the evolution of stress level over time.

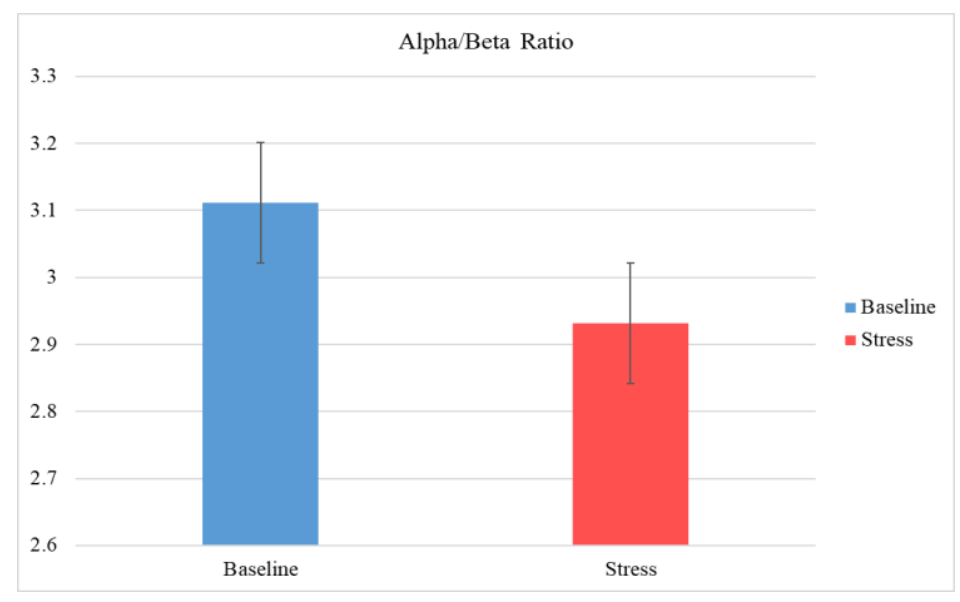

Figure 4. Alpha/Beta ratio before and after exposed to VR stimulus 


\subsection{Theta/Beta Ratio}

The Theta/Beta ratio in Figure 5 as well reveals the value has correlated negatively to stress. Theta oscillations manifest brain activity that regulates motivational and emotional processes [30] and the Theta/Beta ratio facilitates the study of emotional cognitive interactions and the relation to underlying biological functioning [31]. Increased value of the ratio relates to reduced anxious cortical control and greater approach-driven affect. Conversely, declined value of Theta/Beta ratio reflects increased frontal cortical control over subcortical with inhibition-driven affect, higher anxiety and fear which is compatible with Figure 5 as the stress response is associated with negative emotions. Indeed, individual with higher top-down PFC control or better prefrontal brain function able to perform higher inhibitory control in response to stress [32]. Nevertheless, higher Theta/Beta ratio is concluded with higher stress level in the study of [29] which is not aligned with Figure 5. In fact, Putman et. al [33] have studied the relation between the Theta/Beta ratio and the cognitive performance anxiety (CPA) stress-induced changes in PFC-mediated attentional control capacities in healthy individuals. The study concluded that the Theta/Beta ratio reflects individual differences in trait attentional control capacity. Individual with increased ratio indicated inattention, suboptimal attentional control and cognitive executive performance. Therefore in this case, the declined value of Theta/Beta ratio reflects the healthy subjects in the present study have performed stronger capability to concentrate. This result is consistent with the stimulus-driven attention is increased during anxiety and stress states to process related information [34].

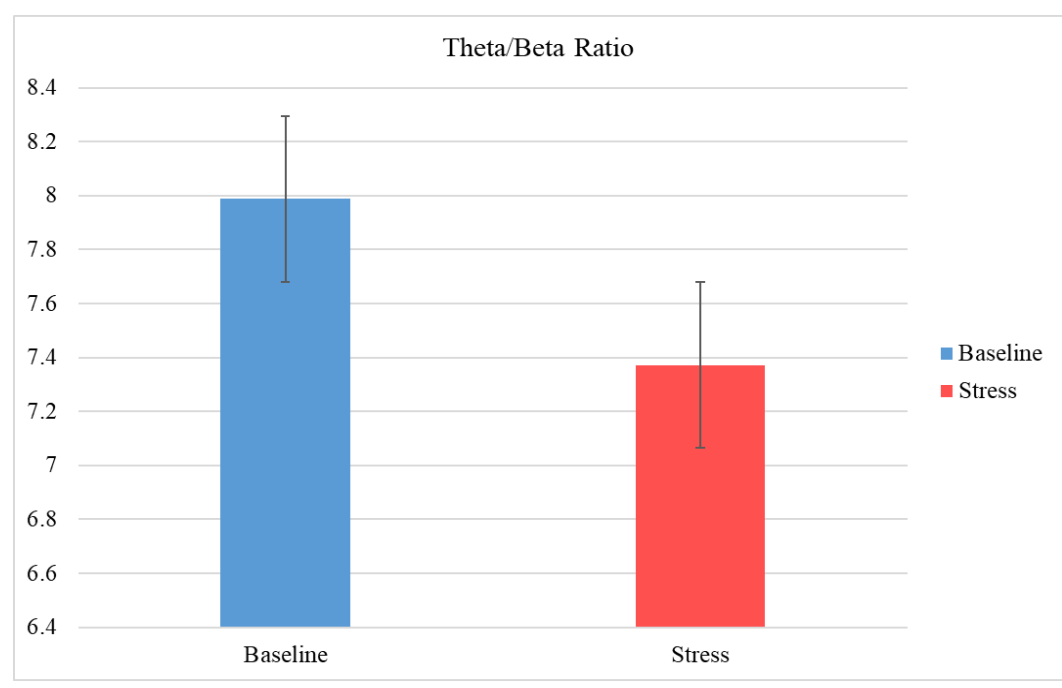

Figure 5. Theta/Beta ratio before and after exposed to VR stimulus

\section{CONCLUSION}

This paper presents an analysis of stress feature by applying the ratio of slow wave over fast wave which are Alpha/Beta ratio and Theta/Beta ratio can be used to reliably discriminate stress from resting state. From the experiment, PSD is computed to recognize the strength and variation of an oscillatory signal within certain frequency ranges. The quantity of power for each frequency bands is extracted and proceed with further analysis which is to calculate the power ratio. Alpha/Beta ratio and Theta/Beta ratio are proven to differentiate the evolution of individual's brain state in response to stress. The declined value of Alpha/Beta ratio after the subjects exposed to stressful condition indicates Beta activity increased due to the variation in response to stress. Whereas, the declined value of Theta/Beta ratio reflects an increase of stimulus-driven attention and the subjects have stronger capability to concentrate. Possibly, Theta/Beta ratio is more suitable to be utilized as an additional predictive qualities in the study of stress and cognitive processing capacity. The changes of mental stress were detectable from EEG data based on PFC in eyes-closed condition and the stress induced by VR stimulus could be discriminated from resting baseline. This study has also validated that the proposed method is able to differentiate between relax and stress state which is potential for the development of stress detection system. The future work will be involved the study to confirm whether the features obtained from Alpha/Beta ratio and Theta/Beta ratio can be used for stress level classification. 


\section{ACKNOWLEDGEMENTS}

The authors would like to thank the Razak Faculty of Technology and Informatics, Universiti Teknologi Malaysia for the sponsorship of the study under research grant no.: R.K130000.7340.4J301 and also to all collaborating partners.

\section{REFERENCES}

[1] C. Rabasa and S. L. Dickson. "Impact of Stress on Metabolism and Energy Balance". Current Opinion in Behavioral Sciences, vol. 9, pp. 71-77, 2016.

[2] S. Ranabir and K. Reetu. "Stress and Hormones". Indian Journal of Endocrinology and Metabolism, vol. 15(1), pp. 18-22, 2011.

[3] I. Kyrou and C. Tsigos. "Stress Hormones: Physiological Stress and Regulation of Metabolism". Current Opinion in Pharmacology, vol. 9(6), pp. 787-793, 2009.

[4] 4. G. M. Slavich and M. R. Irwin. "From Stress to Inflammation and Major Depressive Disorder: A Social Signal Transduction Theory of Depression". Psychological Bulletin, vol. 140(3), pp. 774-815, 2014.

[5] K. B. Koh, J. K. Park, C. H. Kim and S. Cho. "Development of the Stress Response Inventory and Its Application in Clinical Practice". Psychosomatic Medicine, vol. 63(4), pp. 668-678, 2001.

[6] S. Cohen, T. Kamarck and R. Mermelstein. "A Global Measure of Perceived Stress". Journal of Health and Social Behavior, vol. 24(4), pp. 385-396, 1983.

[7] H. Lackschewitz, G. Hüther and B. Kröner-Herwig. "Physiological and Psychological Stress Responses in Adults with Attention-Deficit/Hyperactivity Disorder (ADHD)". Psychoneuroendocrinology, vol. 33(5), pp. 612-624, 2008.

[8] E. Klumbies, D. Braeuer, J. Hoyer and C. Kirschbaum. "The Reaction to Social Stress in Social Phobia: Discordance between Physiological and Subjective Parameters". PLoS ONE, vol. 9(8), pp. e105670, 2014.

[9] M. S. Kidwai and S. H. Saeed. "A Novel Approach for Detection of Neurological Disorders through Electrical Potential Developed in Brain". International Journal of Electrical and Computer Engineering (IJECE), vol. 9(4), pp. 2751-2759, 2019.

[10] M. Mustafa, K. A. A. Nawas, R. Samad, D. Pebrianti and N. R. H. Abdullah. "K-NN Classification of Brain Dominance". International Journal of Electrical and Computer Engineering (IJECE), vol. 8(4), pp. 2494-2502, 2018.

[11] A. H. Jahidin, M. N. Taib, N. M. Tahir and M. S. A. M. Ali. "IQ Classification Via Brainwave Features: Review on Artificial Intelligence Techniques". International Journal of Electrical and Computer Engineering (IJECE), vol. 5(1), pp. 84-91, 2015.

[12] F. P. George, I. Mannafee, P. S. F. Hossain, M. Z. Parvez and J. Uddin. "Recognition of Emotional States Using EEG Signals Based on Time-Frequency Analysis and SVM Classifier". International Journal of Electrical and Computer Engineering (IJECE), vol. 9(2), pp. 1012-1020, 2019.

[13] F. M. Al-Shargie, T. B. Tang, N. Badruddin and M. Kiguchi. "Towards Multilevel Mental Stress Assessment Using Svm with Ecoc: An EEG Approach". Medical \& Biological Engineering \& Computing, vol. 56(1), pp. 125-136, 2018.

[14] R. Khosrowabadi, C. Quek, K. K. Ang, S. W. Tung and M. Heijnen. "A Brain-Computer Interface for Classifying EEG Correlates of Chronic Mental Stress". International Joint Conference on Neural Networks, 2011, pp. 757-762.

[15] X. Hou, Y. Liu, O. Sourina, T. Y. R. Eileen, L. Wang and W. Muller-Wittig. "EEG Based Stress Monitoring". IEEE International Conference on Systems, Man, and Cybernetics, 2015, pp. 3110-3115.

[16] B. B. Purkayastha and K. K. Sarma. "A Digital Phase Locked Loop Based Signal and Symbol Recovery System for Wireless Channel": Springer India; 2015.

[17] M. Teplan. "Fundamental of EEG Measurement". Measurement Science Review, vol. 2(2), pp. 1-11, 2002.

[18] S.-H. Seo and J.-T. Lee. "Stress and EEG". Convergence and Hybrid Information Technologies, 2010, pp. 410-426.

[19] F. M. Al-Shargie, M. Kiguchi, N. Badruddin, S. C. Dass, A. F. M. Hani and T. B. Tang. "Mental Stress Assessment Using Simultaneous Measurement of EEG and fNIRS". Biomedical Optics Express, vol. 7(10), pp. 3882-3898, 2016.

[20] A. F. T. Arnsten. "Stress Signalling Pathways That Impair Prefrontal Cortex Structure and Function". Nature Reviews Neuroscience, vol. 10(6), pp. 410-422, 2009.

[21] N.-H. Liu, C.-Y. Chiang and H.-C. Chu. "Recognizing the Degree of Human Attention Using EEG Signals from Mobile Sensors". Sensors (Basel), vol. 13(8), pp. 10273-10286, 2013.

[22] B. T. Jap, S. Lal, P. Fischer and E. Bekiaris. "Using EEG Spectral Components to Assess Algorithms for Detecting Fatigue". Expert Systems with Applications, vol. 36(2), pp. 2352-2359, 2009.

[23] J. F. Lubar. "Discourse on the Development of EEG Diagnostics and Biofeedback for AttentionDeficit/Hyperactivity Disorders". Biofeedback and Self-Regulation, vol. 16(3), pp. 201-225, 1991.

[24] A. R. Clarke, R. J. Barry, D. Karamacoska and S. J. Johnstone. "The EEG Theta/Beta Ratio: A Marker of Arousal or Cognitive Processing Capacity?". Applied Psychophysiology and Biofeedback, vol. 44(2), pp. 123-129, 2019.

[25] D. Cho, J. Ham, J. Oh, J. Park, S. Kim, N.-K. Lee, et al. "Detection of Stress Levels from Biosignals Measured in Virtual Reality Environments Using a Kernel-Based Extreme Learning Machine". Sensors (Basel), vol. 17(10), pp. 18, 2017. 
[26] G. Huang, J. Meng, D. Zhang and X. Zhu. "Window Function for EEG Power Density Estimation and Its Application in SSVEP Based BCIs". In: S Jeschke, HH Liu,D Schilberg. Intelligent Robotics and Applications. Lecture Notes in Computer Science. 71022011. p. 135-144.

[27] D. P. Subha, P. K. Joseph, R. A. U. and C. M. Lim. "EEG Signal Analysis: A Survey". Journal of Medical Systems, vol. 34(2), pp. 195-212, 2010.

[28] C. Hasegawa and K. Oguri. "The Effects of Specific Musical Stimuli on Driver S Drowsiness". IEEE Intelligent Transportation Systems Conference, 2006, pp. 817-822.

[29] N. Salma, B. Mai, K. Namuduri, R. Mamun, Y. Hashem, H. Takabi, et al.. "Using EEG Signal to Analyze IS Decision Making Cognitive Processes". Information Systems and Neuroscience, 2017, pp. 211-218.

[30] G. G. Knyazev. "Motivation, Emotion, and Their Inhibitory Control Mirrored in Brain Oscillations". Neuroscience \& Biobehavioral Reviews, vol. 31(3), pp. 377-395, 2007.

[31] P. Putman, J. v. Peer, I. Maimari and S. v. d. Werff. "EEG Theta/Beta Ratio in Relation to Fear-Modulated Response-Inhibition, Attentional Control, and Affective Traits". Biological Psychology, vol. 83(2), pp. 73-78, 2010.

[32] L. E. Roos, E. L. Knight, K. G. Beauchamp, E. T. Berkman, K. Faraday, K. Hyslop, et al. "Acute Stress Impairs Inhibitory Control Based on Individual Differences in Parasympathetic Nervous System Activity". Biological Psychology, vol. 125, pp. 58-63, 2017.

[33] P. Putman, B. Verkuil, E. Arias-Garcia, I. Pantazi and C. v. Schie. "EEG Theta/Beta Ratio as a Potential Biomarker for Attentional Control and Resilience against Deleterious Effects of Stress on Attention". Cognitive, Affective, \& Behavioral Neuroscience, vol. 14(2), pp. 782-791, 2014.

[34] J. Sanger, L. Bechtold, D. Schoofs, M. Blaszkewicz and E. Wascher. "The Influence of Acute Stress on Attention Mechanisms and Its Electrophysiological Correlates". Frontiers in Behavioral Neuroscience, vol. 8, pp. 353, 2014.

\section{BIOGRAPHIES OF AUTHORS}

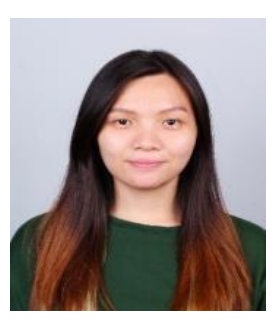

Tee Yi Wen received her Bachelor's Degree in Information Technology (Graphics and Multimedia) from Universiti Tenaga Nasional, Malaysia in 2016; Diploma in Information Technology from Kuala Lumpur Infrastructure University College, Malaysia in 2012.

Currently pursuing her Master's Degree in Razak Faculty of Technology and Informatics, Universiti Teknologi Malaysia, Kuala Lumpur, Malaysia.

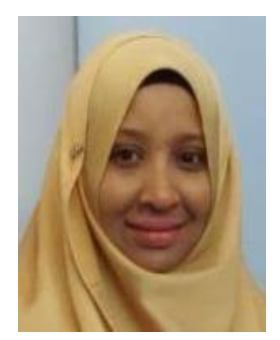

Siti Armiza is from Kuala Lumpur, Malaysia. She received the B.Eng. degree in Electrical Engineering (Microelectronics) from Universiti Teknologi Malaysia in 1998, and the M.Eng. as well as Ph.D degrees in Electrical Engineering from Universiti Teknologi Malaysia in 2001 and Universiti Teknologi MARA in 2016 respectively. She started as a tutor in 1998 and now has become a senior lecturer at Universiti Teknologi Malaysia, Kuala Lumpur. In 2012, she joined the UTM Razak School of Engineering and Advanced Technology as a Lecturer and Researcher, a school that offers undergraduate and postgraduate students from various disciplines. Her current research interests include EEG signal processing, bio-signal processing, psychophysiological interactive tools, and bio-signal monitoring tools. She is a member of IEEE Malaysia Section, IEEE EMBS Malaysia Chapter and IEEE Signal Processing Society Malaysia Chapter. In 2016, her research paper has been recognised by the IEEE WIE and awarded as the best research paper for her outstanding work. 